\title{
A Machine Learning and Cognition-Based Framework for Assessing Academic Performance
}

\author{
Isong, Etebong Bassey \\ Department of Computer Science \\ Akwa Ibom State University \\ Ikot-Akpaden, Nigeria \\ E-mail: etebongisong@aksu.edu.ng
}

\begin{abstract}
The ability to predict future occurrences creates a platform to ensure that these occurrences or expected outcomes happen by design and in the process enabling better decision making and management especially in the field of learning. Machine learning models, which has the advantage of being trained, affords the opportunity of predicting, forecasting and modeling phenomena to ascertain conformance to desired standards and taking necessary decisions based on the outcome. This paper elucidates Artificial Neural Networks (ANN) as a machine learning algorithm that can be harnessed to develop a system for evaluating Students' Academic Performance. The operational frameworks were elucidated and possibilities outlined.
\end{abstract}

Keywords: Students, Academic, Performance, Evaluation, AAN, Learning and Systems.

Aims Research Journal Reference Format:

Isong, E. (2018): A Machine Learning and Cognition-Based Framework for Assessing Academic Performance

Advances in Multidisciplinary Research Journal. Vol. 4. No. 4, Pp 85-90

Article DOI: dx.doi.org/10.22624/AIMS/V5N1P8

\section{INTRODUCTION}

Evaluation of students' performances are based on certain parameters that are necessary yardstick and tool for measuring the effectiveness of students in a number of selected academic programs or specified tasks requiring skills as depends to a great extent on tests much more closely related to the specific task of the interest. These tests could range from examinations based on taught lessons to cognitive abilities of the subjects. Since there is yet to be a universally accepted scientific definition of intelligence or theories to measure intelligence, the purpose of these evaluations is reduced to predicting future performances of the students being tested based on their individual ability to perform as required in an academic program or in a skilled work task (Fischler \& Firschein, 1987).

Grade Point Average (GPA), being a traditional statistical method has been considered by numerous studies as the best summary of student learning because of its strong prediction of performance (e.g. for each particular level of education which informs academic administrators if a student is to be promoted to the next level or not. The GPA is also agreed to be an effective tool in measuring future life productivity of the student in terms of income earned as well as job performance. Whereas the Grade Point Average (GPA) has been touted as the basis for performance evaluation by some studies there are others who believe that this falls short of adequate evaluation citing cognitive and non-cognitive measures as a better alternative Musso, Eva, Eduardo \& Filip (2013). It can be deduced therefore from the ongoing arguments, that scholars and researchers are divided on the evaluation methods that should be adopted over the others. Despite the efforts of the Nigerian government, educational managers and administrators across the different levels of education, poor academic performances still remain on the ascendency and this creates persistent problems (Dursun, 2010) to the very essence of education which is applicative learning.Poor performance denotes performing below the set standards of an academic institution. This concept of poor performance is relative as it varies across disciplines or fields and across institutions. Performances generally affect University rankings, school reputation and financial wellbeing (Dursun, 2010). 


\section{RELATED WORKS}

Musso et al. (2013) employed a new methodological approach for the field of learning and education. Although this approach had been widely applied in areas such as computational science, engineering and economics, it was yet to be applied to academic evaluation. They used cognitive and non-cognitive measures of students, together with background information to design predictive models of students' performance using Artificial Neural Networks (ANN). These predictions constituted a true predictive classification of academic performance over a period of one year in advance of the actual observed measure of academic performance.

Using a total sample of 864 university students (split into three levels of General Academic Performance (GAP); low, mid and high measured by grade-point-average of both genders, ages ranging from 18 to 25 years, they developed three neural network models that used a back propagation multilayer perceptron composed of nonlinear units. Two of the models reached a precision of $100 \%$ identifying the top $33 \%$ and lowest $33 \%$ groups respectively. The third model reached precisions from $87 \%$ to $100 \%$ identifying low, mid and high performance levels for the three groups. The result when compared to those of traditional methods such as Discriminant Analysis demonstrated greater accuracy.

Oladokun, Adebanjo \& Charles-Owoba (2008) built a neural network based on the Multilayer Perceptron with two hidden layers and five processing elements per layer. The network predicted accurately 9 out of 11 of candidates (good data) with either 1st Class or 2nd Class upper, 8 out of 15 of candidates (average data) with a 2nd Class lower and 7 out of 8 of candidates (poor data) with either a 3rd Class or Pass thus demonstrating an accuracy of $82 \%$ for the Good, $53 \%$ for the Average and $88 \%$ for the Poor Classification. This indicated an accuracy of about $74 \%$ for the artificial neural network and compared to other traditional methods displayed the potential efficacy of the ANN models as a predictive tool.

Raheela, Merceron \& Pathan (2015) applied several predicting methods on data sets (of about 347 students broken into two data sets) containing students' pre-admission data and examination scores of first and second academic year courses in predicting the students' performance at the end of the degree. The recorded an accuracy of $83.65 \%$ on data set two which had more data entries using Naïve Bayes algorithm while Artificial Neural Network recorded an accuracy of $62.50 \%$. However, since the results of the Naïve Bayes were not easy to interpret it was termed as not actionable.

Usman \& Adenubi (2013) used Artificial Neural Networks (ANNs) to develop a model for predicting the final grade of a University student before graduating such student employing data consisting thirty (30) randomly selected students in the department of Computer Science, who have completed four academic sessions in the University. They trained and simulated the Artificial Neural Network models using the nntool of MATLAB (2008a) software. From the test data evaluation obtained, the ANN model correctly predicted the final grade of the students to an accuracy of $92.7 \%$.

\section{MACHINE LEARNING FRAMEWORK}

\subsection{Artificial Neural Networks (ANN)}

Many research materials abound on the subject of the Artificial Neural Network (ANN) model which has been touted by various researchers as a more effective tool in performance prediction and forecasting behavior of irregular data sets. An Artificial Neural Network (ANN), which is motivated by research into the configuration and functionality of the brain (Atiya, 1991), has gained a lot of interest from researchers within the last decade. According to Sumam, (2016) in his work, an Artificial Neural Network, also called Neural Network (NN), is a model of computation motivated from their biological counterparts. It is a computerized simulation and representation of the Human Neural System. 
The neural network is a combination of interconnected groups of artificial neurons processing information via a connectionist methodology to computation. In practical application, neural networks are applied to data sets that defy linear statistical modeling tools as such neural networks can model complex relationships between inputs and outputs as well as find patterns in the given data sets. Basically, since Artificial Neural Networks require vast collections of data, they are best applied where there is a data warehouse. Neural Networks Models are applied using a number of architectures based on their methods of processing data. The most popular and most used of this architectures is considered below.

\subsection{Feed-Forward (Multilayer Perceptron) Neural Networks}

Feed forward (multilayer perceptron) neural networks have been extensively dealt with in the neutral literature as outlined by Lippmann's review and Wasserman's paper. They are backpropagation networks that only allows a unidirectional flow of signal from the input layer to the output layer without the presence of feedback mechanisms or loops as a result, information only moves forward, from the input to the output nodes (Kalejaye, Folorunso \& Usman (2015). These networks can be trained using a set of data called the training set which consist of an input data and a corresponding output data.

Sumam submits that the Multilayer Perceptron (MLP) has over the past decade been the most used of all neural network architectures (Sumam, 2016). Some characteristics associated with this architecture include:

i. The presence of any number of input neurons as desired.

ii. The presence of hidden layers ranging from one to many with each layer having as many neurons as desired.

iii. The hidden layers of the MLP employs a number sigmoid activation functions in solving problems.

iv. There are connections linking the input layer to the hidden layers and the hidden layers in turn link to the output layer in strictly one direction of flow.

As outlined above we can deduce that the feed-forward network consists of several layers that are interconnected together. In this network, the last layer is called the output or visible layer and the other layers are called the hidden layers. Most applications usually use one or two hidden layers. The problems to be solved determine the number of neurons employed per hidden layer of the network. However, it takes trail and errors to specify this and the more difficult the problem the larger the required sizes of the hidden layers. The data processing can extend over multiple (layers of) units, but no feedback connections are present i.e., connections extending from outputs units to inputs units in the same layer or previous layers. This is achieved by some advanced mathematical definitions where; $y_{i}^{[1]}$ denotes the output of the $i^{\text {th }}$ neuron of layer $t \cdot G_{i}^{(0) y}$ denotes the $i^{\text {th }}$ input to the network).

The function of the network is defined as;

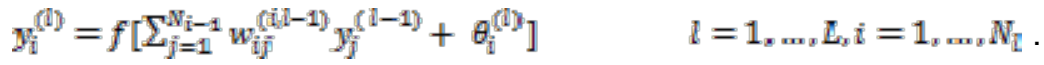

Where $w_{i j}^{(i j-1)}$ denotes the weight from neuron $j$ of layer $l-1$ to neuron $i$ of layer $l_{t} \theta_{i j}^{(\mathbb{D})}$ is the threshold of neuron $i$ of layer $l$. The function $f$ is taken as a unit step or a sign function or as a sigmond-shaped function, example: $f(x)=\tanh (x)$ or $f(x)=\frac{1}{1+\varepsilon^{-x}}$. The multilayer perceptron or feed-forward network is diagrammatically illustrated below. 


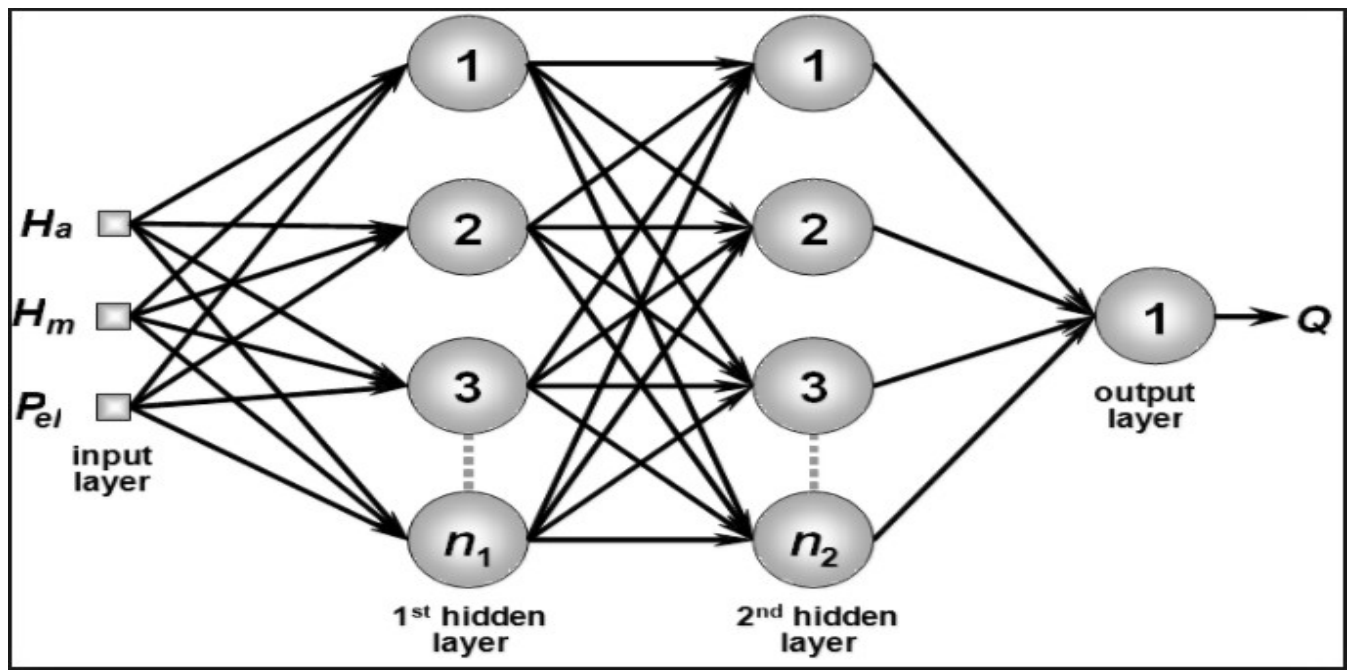

Fig 1: Multilayer Perceptron

Source: (da Silva I. N., Cagnon J. A. \& Saggiaro J. N. 2013)

\section{COGNITION FRAMEWORK}

The uniqueness of the human brain, its capacities, functionalities and processing power, which can be learned from studying Artificial Neural Networks, opens an avenue to better appreciate the immense abilities within man and discover new ways of solving problems using intelligent systems. So there is need to develop system for predicting and forecasting which will assist the students and education managers in making informed decisions.

Some studies have indicated that there exists a strong correlation between the cognitive abilities of students and academic performances (Musso et al. 2013). It has been observed that cognitive make up of students has a very strong relationship with their academic achievements (Colom, Escorial, Chin Shih, \& Privado, 2007 cited in Musso et al. (2013). Yet again, scholars are divided on the subject of applying cognitive measures to performance evaluation. It is argued that considering students' cognitive abilities can result to a relatively strong performance evaluation (Colom et al., 2007 cited in M. F. Musso et al. 2013). Importantly, there could be a stronger evaluation of academic performances using both background and non-cognitive predictors (Musso et al. 2013). It can be seen from the argument that evaluation of general academic performances would be better done if both cognitive and non-cognitive factors as well as background data are considered as predictors. Furthermore, student appraisal would fall short unless all the above factors are taken aboard.

\subsection{Concentration and Academic Performance}

Studies have proven that the human cognitive system is at every instant of time being bombarded by a plethora of inputs from the environment all seeking to be given attention and concentration (Musso et al. 2013). Since human cannot concentrate optimally on several of these bombardments simultaneously due to the limitedness of the cognitive system academic performances tend to be poor where the concentration of the student is divided. Studies have found that working memory capacity has a strong relation with concentration (Redick \& Engle, 2006). The importance of concentration in relation to academic performance cannot be relegated if better performances are desired (Gsanger, Homack, Siekierski \& Ricco, 2002; Jimmerson, Dubrow, Adam, Gunnar \& Bozoky, 2006). 


\subsection{Social Media And Academic Performance}

It is an established fact that Social media has come to stay and has gained worldwide acceptance (Al Rahmi \& Othman, 2013). Although social media serves very vital needs of providing affordable communication especially among University students on the one hand but on the other it has been found by studies to be one of the most prevalent factors encouraging poor academic performances among students. This is because time supposed to be used for studies is spent on social media.

Al-Rahmi \& Othman (2016) pointed out that Facebook users devoted lesser time to their studies in comparison to non-users and subsequently had lower GPAs. There is a further mention that among various unique distractions of every single generation, Facebook remains a major distraction of current generation (Al-Rahmi \& Othman, 2016; Karpinski A., 2009; Karpinski, \& Duberstein, 2009).

There is a poor performance and overall influence when the media is overused in a way that does not academically improve learning (Al Rahmi \& Othman, 2013).

\subsection{Passion and Academic Performance}

It is an accepted concept from experience that passion displayed via commitment and persistence is a vital virtue required for better academic performance. It is often seen from a theoretical point of view that commitment and perseverance are features of well performing students and are often lacking in those that perform poorly.

This concept had been conceptualized systematically by Duckworth, Peterson, Matthews \& Kelly (2007). They propose that two dimensions characterize passion or tenacity: perseverance of effort (striving harder to accomplish goals despite the hard-ships faced) and commitment or consistency of interests (showing steady interest over time).

\section{CONCLUSION}

This research has demonstrated the efficiency and effectiveness of an Artificial Neural Network in academic performance evaluation. Student Intelligent Quotient (Common Sense) when combined with their attention in class as well as class attendance is a factor that influences the performances of students. The mental capacity of a student can be improved by conscious mental exercises such as engaging in creative and logical thinking, mental calculations as well as taking on challenging IQ test questions.

\subsection{Suggestion For Future Research}

Future works may seek to analyze an Artificial Neural Network and its functionalities as well as discuss some of the factors that should be considered in evaluating a student performance. Similarly, research efforts can be directed at using student's cognitive ability (IQ rating) as one of the variables and yardstick for evaluating performance. 


\section{REFERENCES}

1. Al Rahmi W. M. \& Othman M. S. (2013). The impact of social media use on academic performance among university students: a pilot study. Journal of Information Systems Research \& Innovation, 1-10. Retrieved from http://seminar.utmspace.edu.my/jisri/.

2. Atiya, Amir (1991). Learning Algorithms for Neural Networks (Doctoral dissertation). Retrieved from California Institute of Technology Pasadena database.

3. Colom, R., Escorial, S., Shih, P.C., Privado, J.: Fluid intelligence, memory span, and ... Personality and Individual Differences 42(8), 1503-1514

4. Dursun Delen (2010). A comparative analysis of machine learning techniques for student retention management. Elsevier International Journal of Forecasting, 49, 498-506.

5. Fischer, M. A. \& Fischein O. (1987): Intelligence: The Eye, the Brain and the Computer. AddisonWesley, 1987. ISBN: 0201120011

6. Gsanger, K., Wong, S., Homack, S., Siekierski, B.,\& Riccio, C. (October, 2002). The relation of memory and attention to academic achievement in children. Presented at the annual meeting of the National Academy of Neuropsychology, Miami, Florida

7. Jimmerson, S. R., Dubrow, E. H., Adam, E., Gunnar, M., \& Bozoky, I. K. (2006).Associations among academic achievement, attention, and andrenocortical reactivity in Caribbean village children. Canadian Journal of School Psychology, 21,120-138

8. Kalejaye B. A., Folorunso O. \& Usman O. L. (2015). Predicting students' grade scores using training functions of artificial neural network. Journal of Natural Science, Engineering \& Technology, 14(1), 25-42.

9. Karpinski, A, \& Duberstein. (2009). A description of Facebook use and academic performance among undergraduate and graduate students. Annual Meeting of the American Educational Research Association, San Diego, California.

10. Musso F. M., Eva K., Eduardo C. C. \& Filip D. (2013). Predicting general academic performance and identifying the differential contribution of participating variables using artificial neural networks. Frontline Learning Research, 1, $42-71$.

11. Oladokun V. O., Adebanjo A. T. \& Charles-Owoba O. E. (2008). Predicting students' performance using artificial neural network: A case study of an Engineering course. The Pacific Journal of Science \& Technology, 9(1),72-79.

12. Raheela A., Merceron A., Pathan M. K. (2015). Predicting student academic performance at degree level: a case study. International Journal of Intelligent Systems and Applications (IJISA), 7(1), 49-61. DOI: 10.5815/ijisa.2015.01.05

13. Sumam S. (2016). Performance evaluation by artificial neural network using WEKA. International Research Journal of Engineering \&Technology (IRJET), 03, 1459 - 1464.

14. Usman O. L. \& Adenubi A. O. (2013). Artificial neural network (ANN) model for predicting students' academic performance. Journal of Science and Information Technology, 1(2), 23-37 